\title{
Pathogenesis of intracranial aneurysm is mediated by proinflammatory cytokine TNFA and IFNG and through stochastic regulation of IL10 and TGFB1 by comorbid factors
}

Sanish Sathyan ${ }^{1}$, Linda V. Koshy', Lekshmi Srinivas', H. V. Easwer², S. Premkumar ${ }^{3}$, Suresh Nair ${ }^{2}$, R. N. Bhattacharya², Jacob P. Alapatt ${ }^{3}$ and Moinak Banerjee ${ }^{1 *}$

\begin{abstract}
Background: Intracranial aneurysm (IA) is often asymptomatic until the time of rupture resulting in subarachnoid hemorrhage $(\mathrm{SAH})$.There is no precise biochemical or phenotype marker for diagnosis of aneurysm. Environmental risk factors that associate with IA can result in modifying the effect of inherited genetic factors and thereby increase the susceptibility to SAH. In addition subsequent to aneurismal rupture, the nature and quantum of inflammatory response might be critical for repair. Therefore, genetic liability to inflammatory response caused by polymorphisms in cytokine genes might be the common denominator for gene and environment in the development of aneurysm and complications associated with rupture.

Methods: Functionally relevant polymorphisms in the pro- and anti-inflammatory cytokine genes IL-1 complex $(I L 1 A, I L 1 B$, and ILIRN), TNFA, IFNG, IL3, IL6, ILI2B, ILIRN, TGFB1, IL4, and IL10] were screened in radiologically confirmed 220 IA patients and 250 controls from genetically stratified Malayalam-speaking Dravidian ethnic population of south India. Subgroup analyses with genetic and environmental variables were also carried out.

Results: Pro-inflammatory cytokines TNFA rs361525, IFNG rs2069718, and anti-inflammatory cytokine IL10 rs1800871 and rs 1800872 were found to be significantly associated with IA, independent of epidemiological factors. TGFB1 rs1800469 polymorphism was observed to be associated with IA through co-modifying factors such as hypertension and gender. Functional prediction of all the associated SNPS of TNFA, IL10, and TGFB1 indicates their potential role in transcriptional regulation. Meta-analysis further reiterates that $I L 1$ gene cluster and IL6 were not associated with IA.

Conclusions: The study suggests that chronic exposure to inflammatory response mediated by genetic variants in pro-inflammatory cytokines TNFA and IFNG could be a primary event, while stochastic regulation of ILIO and TGFB1 response mediated by comorbid factors such as hypertension may augment the pathogenesis of IA through vascular matrix degradation. The implication and interaction of these genetic variants under a specific environmental background will help us identify the resultant phenotypic variation in the pathogenesis of intracranial aneurysm. Identifying genetic risk factors for inflammation might also help in understanding and addressing the posttraumatic complications following the aneurismal rupture.
\end{abstract}

Keywords: Inflammation, Cytokine, Aneurysm, SNP, TNFA, IL10, TGFB1, Meta-analysis

\footnotetext{
* Correspondence: mbanerjee@rgcb.res.in

${ }^{1}$ Human Molecular Genetics Laboratory, Rajiv Gandhi Centre for

Biotechnology, Thiruvananthapuram 695014Kerala, India

Full list of author information is available at the end of the article
} 


\section{Introduction}

Intracranial aneurysm (IA) is often asymptomatic until the time of rupture resulting in subarachnoid hemorrhage (SAH).There is no precise biochemical or phenotype marker for diagnosis of aneurysm. Rupture of cerebral aneurysm is the foremost cause for spontaneous subarachnoid hemorrhage (SAH). Intracranial aneurysm accounts for $85 \%$ of subarachnoid hemorrhage (SAH), which contributes for 5-15\% of strokes [1] but occurs at a fairly young age and accounts for $25 \%$ of stroke-related mortality [2]. In an earlier study, we have reported that hypertension and smoking are causal risk factors which might also modify the effect of genetic factors resulting in increased susceptibility to aSAH in the Indian population [3]. Inherited and acquired risk factors indicate influence on hemodynamic factors, defects in the vessel wall which may be in the muscular layer (tunica muscularis) of arteries, or alterations in the internal elastic membrane (lamina elastica interna) of cerebral arteries [4]. It is believed that hemodynamic stress can trigger localized inflammatory infiltration leading to formation of intracranial aneurysm by thinning and weakening in intracranial vessel [5]. Studies have pointed towards the presence of inflammatory cells such as macrophages, $\mathrm{T}$ and $\mathrm{B}$ lymphocytes in the wall of intracranial arteries [6].Various expression studies carried out till date with respect to intracranial aneurysm, support the role of inflammation and immune response with intracranial aneurysm [7]. These reports clearly demonstrate that inflammation plays a pivotal role in the pathogenesis of intracranial aneurysm.

Inflammation is an essential component of the immune response which involves inflammatory factors such as cytokines. When endothelial cells undergo inflammatory activation, it recruits cytokines, growth factors, and matrix metalloproteinase's (MMPs). Balance between pro-inflammatory and anti-inflammatory cytokines defines the control of inflammation [8]. If vascular inflammation progresses unresolved, it can lead to vascular disease, whereas delayed inflammatory response can lead to ECM deposition, granular tissue formation and connective tissue growth. One would presume that genetic variability in cytokine genes can result in differential activation of immune response. The present study intends to explore the genetic role of pro-inflammatory and anti-inflammatory cytokine genes in intracranial aneurysm in a south Indian population. The study intends to evaluate the functionally relevant polymorphic variants of pro-inflammatory and antiinflammatory cytokine genes [IL-1 complex (IL1A, IL1B, and IL1RN), TNFA, IFNG, IL3, IL6, IL12B, IL1RN, TGFB1, IL4, and IL1O] and their role in aSAH. The role of these cytokine variants in influencing the epidemiological risk factors such as hypertension, gender and smoking and its relevance in global perspective will also be examined in the present study.

\section{Materials and methods Study population}

The study populations consist of radiologically confirmed 220 IA cases and 250 ethnically and age-matched controls from the Malayalam-speaking Dravidian ethnic population of south India. The study was restricted to Malayalamspeaking Dravidian ethnic population of south India to avoid population stratification issues [9]. Patients having saccular intracranial aneurysm with aSAH were initially screened by $\mathrm{CT}$ and were angiographically confirmed by magnetic resonance angiography (MRA) and digital subtraction angiography (DSA). Exclusion criteria include patient with non-saccular aneurysm, arteriovenous malformation (AVMs), and other hereditary connective tissue disorders like autosomal dominant polycystic kidney disease, Marfan syndrome, and Ehlers-Danlos syndrome. Cases were recruited from two main tertiary care neurosurgical centers in Kerala. All the patients were rated based on WFNS scale. The control population consists of age-, sex-, and ethnicity-matched individuals who were symptomatically normal and did not possess any symptoms or family history of intracranial aneurysm. All the study subjects gave informed, written consent in a standard consent form to participate in the study after being provided with and receiving a full explanation of study protocols and objectives. The present study was approved by the institutional ethics committee of Rajiv Gandhi Center for Biotechnology, established as per the Indian council of medical research guidelines. Peripheral blood was collected from the study subjects in EDTA-coated vials, and DNA was isolated by conventional phenol-chloroform method.

\section{SNP selection and genotyping}

SNPs were selected based on the functional relevance and minor allele frequency using genotype data obtained from Caucasian individuals in the HapMap project (HapMap Data Rel 24/Phase II Nov08, on NCBI B36 assembly, dbSNP b126). Some of the SNPs of IL6, IL12B, IL1A, and $I L 1 B$ were selected based on their associations in the previous studies. Genotyping of 22 SNPs from (IL1A, IL1B, and IL1RN), TNFA, IFNG, IL3, IL6, IL12B, IL1RN, TGFB1, IL4, and IL10 was carried out using TaqMan allelic discrimination assays (Applied Biosystems, Foster City, CA, USA) and KASPar method (KBioscience, Hoddesdon, UK) (Additional file 1: Table S1). TaqMan and KASPar reactions were performed in a 5- $\mu$ l volume according to the manufacturer's instructions on a 384-well-based ABI7900HT thermo cycler. Detection was performed using an ABI PRISM 7900 HT sequence detection system with SDS 2.4 software (Applied Biosystems, Foster City, CA, USA).

\section{Statistical analysis}

Genotype and allelic frequencies were computed and were tested for deviation from Hardy-Weinberg equilibrium 
(ihg2.helmholtz-muenchen.de/cgi-bin/hw/hwa1.pl). All statistical analyses were performed using the GraphPad Prism 5.01, San Diego, CA, USA. We considered $p$ value of $<0.05$ as significant. Chi-square test, allelic odds ratios (OR), and $95 \%$ confidence intervals (CI) were calculated by Fisher's exact test (two-tailed). To estimate linkage disequilibrium (LD) between pairs of loci in the patient and control populations, standardized disequilibrium coefficient $\left(D^{\prime}\right)$ and squared correlation coefficient $\left(r^{2}\right)$ were calculated using Haploview 4.1 (www.broad.mit.edu/mpg/ haploview/). LD blocks were defined in accordance with Gabriel's criteria. Further stratification of the patients was done to understand the role of associated cytokine gene variant between genders and hypertensive and nonhypertensive groups.

Functional prediction of the deleterious effect if any of the associated SNPs with respect to the functional categories such as protein coding, splicing regulation, transcriptional regulation, and post-translation was assessed in silico using F-SNP program (compbio.cs.queensu.ca/F$\mathrm{SNP} /$ ), FastSNP (fastsnp.ibms.sinica.edu.tw), SNPNexus (snpnexus.org), HaploReg (broadinstitute.org/mammals/ haploreg), and regulomeDB (regulome.stanford.edu). FSNP extracts information from large number of resources such as PolyPhen, SIFT, SNPeffect, SNPs3D, LS-SNP, Ensembl, ESEfinder, RescueESE, ESRSearch, PESX, TFSearch, Consite, GoldenPath, KinasePhos, OGPET, and Sulfinator to generate a functional significance (FS) score.

A meta-analysis with the random effects and fixed effects model was performed for previously studied SNPs using Review Manager 5.2 (reviewmanager.software. informer.com/5.2/). All the previous studies which explored similar variants in genes involved in inflammatory and anti-inflammatory cytokines were included in this study. The meta-analysis included the most studied SNPs in immune response, rs1800796 of IL6 [10-14], rs3212227 of $I L 12 B$ [15], and rs16944 of $I L 1 B[16,17]$ (Additional file 1: Table S2). The inconsistency index $I^{2}$ was used to assess between-study heterogeneity. A $p$ value of $<0.05$ was considered as significant throughout the analyses.

\section{Results}

The demographic and clinical characteristics of subjects were recorded and are summarized in Table 1. All the studied variants were in Hardy-Weinberg equilibrium $(p>0.05)$ in healthy controls. Using a case control design, we observed a novel association with pro-inflammatory cytokines TNFA and IFNG (Table 2, Fig. 1). In TNFA rs361525 was found to be associated with IA at allelic ( $p=$ 0.019 , OR 1.89 , CI 1.16 to 3.06 ) and genotypic level ( $p=$ $0.010)$. In case of IFNG, of the two variants screened (rs2069718 and rs2430561), a genotypic association was observed for rs2069718 $(p=0.020)$. However, some of the
Table 1 Clinical characteristics of patients

\begin{tabular}{ll}
\hline Patient characteristics & \\
Mean age \pm SD, years & $51.17 \pm 11.37$ \\
Men, \% & 55.7 \\
Women, \% & 44.2 \\
History of hypertension,\% & 35 \\
History of diabetes, \% & 5.4 \\
Family history of aSAH, \% & 3.7 \\
Cigarette smoking, \% & 42.92 \\
Alcohol use, \% & 21.8 \\
Intracranial aneurysm location & Percentage \\
Anterior communicating artery & 39.21 \\
Anterior cerebral artery & 8.37 \\
Middle cerebral arteries & 22.91 \\
Internal carotid artery & 17.18 \\
Posterior communicating artery & 8.81 \\
Basilar artery & 2.64 \\
Posterior cerebral artery & 0.44 \\
Vertebral arteries & 0.44 \\
\hline
\end{tabular}

extensively studied pro-inflammatory cytokine variants such as rs1800587of IL1A and rs1143627 and rs16944 of $I L 1 B$ in $I L 1$ gene cluster were not found to be associated with IA in south Indian population (Additional file 1: Table S3). Similarly with IL6 variants too, we could not observe a major significant association although IL6 variants rs1800796 (-572C/G) indicated a trend towards association (Table 2) with IA in the present study population. The SNPs in pleiotropic cytokine genes IL3, IL12B, IL4, and anti-inflammatory gene TGFB1 were also not found to be associated with intracranial aneurysm (Additional file 1: Table S3). However, two linked SNPs of anti-inflammatory cytokine IL10 rs1800871 and rs1800872 indicated an association with heterozygous genotype with $p$ value of 0.016 and 0.005 , respectively, with IA (Table 2). The frequency of these variants vary widely across different world population. In silico functional prediction results for all the associated SNPs indicate that TNFA rs361525 and IL10 rs1800871 and rs1800872 have potential role in transcriptional regulation (Table 2, Additional file 1: Table S4).

Stratification of data based on hypertension and gender was further carried out within the patient group to identify whether genetic association for IA was influenced by hypertension or gender or independent of these factors. We could not find any significant association for this stratification analysis with exception to TGFB1 Arg25Pro rs1800471. The SNPs that were associated with IA were not influenced by genetic association of hypertension (Fig. 2) or gender (Additional file 1: Figure S1). Interestingly, in the subgroup analysis, TGFB1 rs1800471 which 
Table 2 Comparison of the genotype and allele frequencies of associated cytokine gene variants between patients and controls

\begin{tabular}{|c|c|c|c|c|c|c|c|c|c|c|}
\hline & & & & & $p$ value & & & $\mathrm{OR}(95 \% \mathrm{Cl})$ & $p$ value & FS score \\
\hline IFNG & & $\mathrm{AA}$ & $A G$ & GG & & A & G & & & \\
\hline \multirow[t]{4}{*}{ rs2069718 } & Cases & 79 & 103 & 39 & 0.020 & 261 & 181 & $1.20(0.926$ to 1.560$)$ & 0.184 & ND \\
\hline & & 0.36 & 0.47 & 0.18 & & 0.59 & 0.41 & & & \\
\hline & Controls & 61 & 143 & 39 & & 265 & 221 & & & \\
\hline & & 0.25 & 0.59 & 0.16 & & 0.55 & 0.45 & & & \\
\hline TNF & & GG & $A G$ & $\mathrm{AA}$ & & G & A & & & \\
\hline \multirow[t]{4}{*}{ rs361525 } & Cases & 171 & 42 & 2 & & 384 & 46 & 1.89 (1.163 to 3.064$)$ & 0.010 & 0.208 \\
\hline & & 0.80 & 0.20 & 0.01 & 0.019 & 0.89 & 0.11 & & & \\
\hline & Controls & 216 & 25 & 2 & & 457 & 29 & & & \\
\hline & & 0.89 & 0.10 & 0.01 & & 0.94 & 0.06 & & & \\
\hline IL6 & & $\mathrm{GG}$ & GC & CC & & G & C & & & \\
\hline \multirow[t]{4}{*}{ rs1800796 } & Cases & 57 & 126 & 37 & 0.050 & 240 & 200 & $0.94(0.729$ to 1.225$)$ & 0.691 & 0.208 \\
\hline & & 0.26 & 0.57 & 0.17 & & 0.55 & 0.45 & & & \\
\hline & Controls & 81 & 111 & 52 & & 273 & 215 & & & \\
\hline & & 0.33 & 0.45 & 0.21 & & 0.56 & 0.44 & & & \\
\hline IL10 & & CC & $\mathrm{CT}$ & $\pi$ & & C & $\mathrm{T}$ & & & \\
\hline \multirow[t]{4}{*}{ rs1800871 } & Cases & 48 & 142 & 40 & 0.016 & 238 & 222 & 1.08 (0.837 to 1.396$)$ & 0.558 & 0.101 \\
\hline & & 0.21 & 0.62 & 0.17 & & 0.52 & 0.48 & & & \\
\hline & Controls & 61 & 118 & 62 & & 240 & 242 & & & \\
\hline & & 0.25 & 0.49 & 0.26 & & 0.50 & 0.50 & & & \\
\hline IL10 & & $\mathrm{AA}$ & $A C$ & CC & & A & C & & & \\
\hline \multirow[t]{4}{*}{ rs1800872 } & Cases & 39 & 143 & 48 & 0.005 & 221 & 239 & $0.955(0.740$ to 1.233$)$ & 0.745 & 0.101 \\
\hline & & 0.17 & 0.62 & 0.21 & & 0.48 & 0.52 & & & \\
\hline & Controls & 62 & 116 & 66 & & 240 & 248 & & & \\
\hline & & 0.25 & 0.48 & 0.27 & & 0.49 & 0.51 & & & \\
\hline
\end{tabular}

FS score functional significance score, $N D$ not determined

was not associated with disease alone was found to be associated with hypertension-mediated resultant IA (Fig. 2).

Meta-analysis with the SNPs in the cytokine genes was carried out with data from earlier studies and including the present study. Meta-analysis with the promoter polymorphism rs1800796 (-572C/G) of IL6 indicated high heterogeneity between studies $\left(I^{2}=93 \%\right)$ which accounts for the large variation in different studies shown by opposite alleles being associated in different world population. This prompted us to carry out meta-analysis using both random and fixed effect models. While comparing $\mathrm{C}$ versus $\mathrm{G}$ allele between IA patients and normal

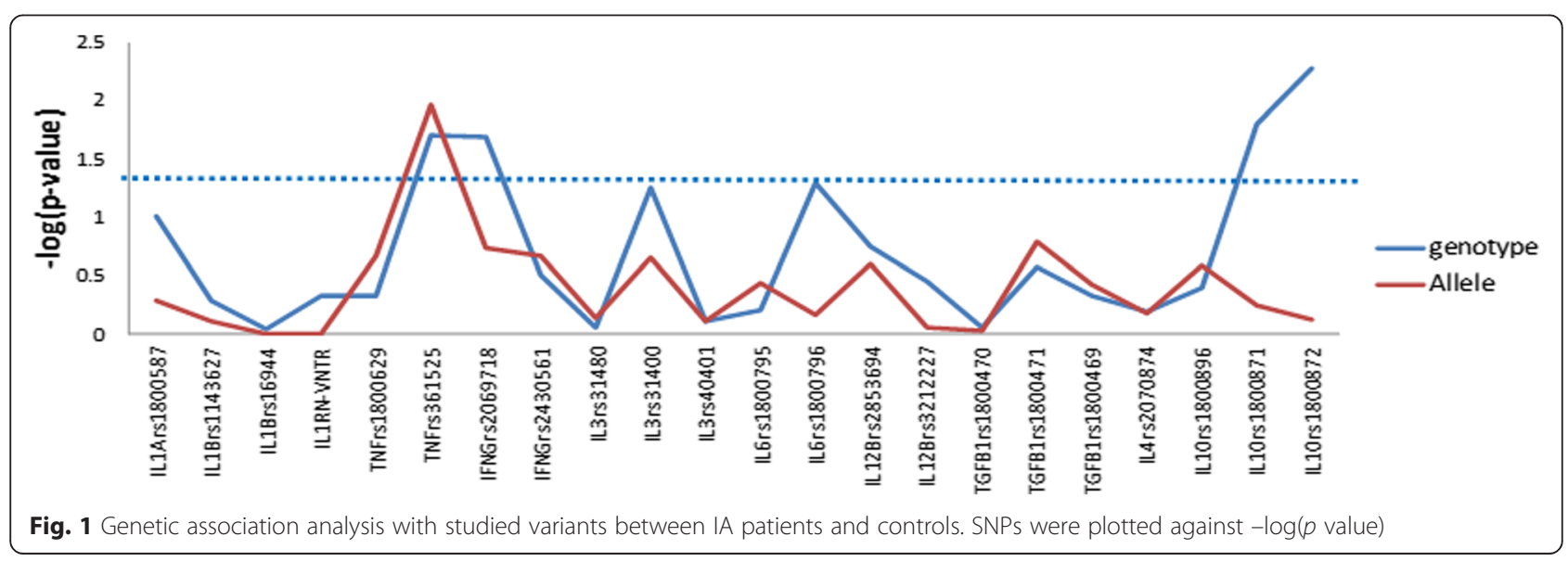




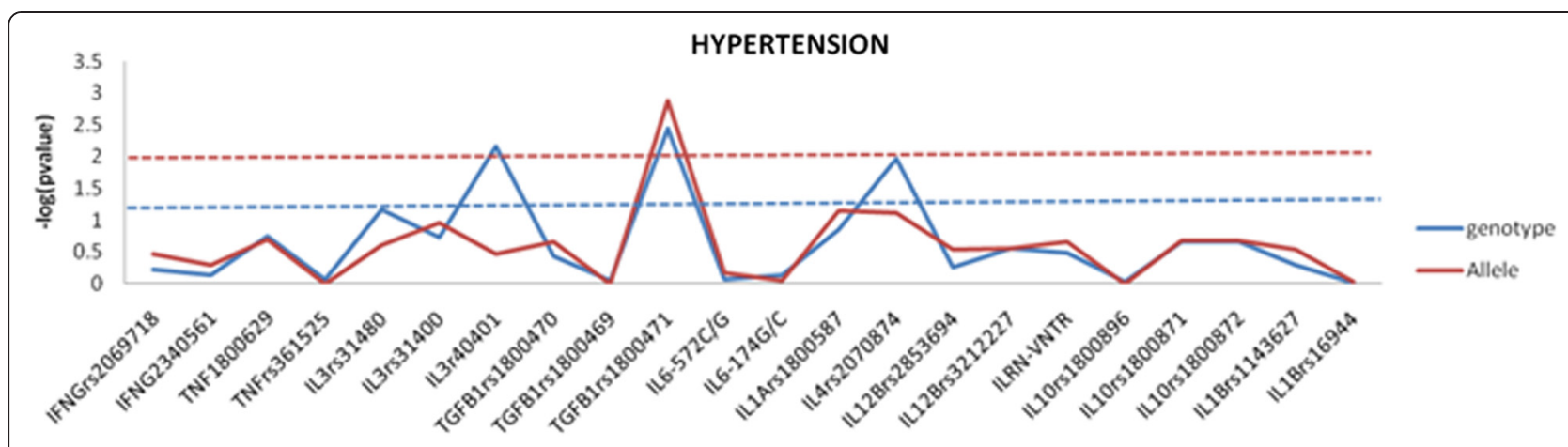

Fig. 2 Genetic association analysis with studied variants between hypertensive and non-hypertensive IA. SNPs were plotted against -log( $p$ value)

control, significant association was observed in fixed effect model $(Z=3.57, p=0.0004)$ while no association was observed in random effect model $(Z=0.02, p=0.98)$ (Fig. 3). Further stratification of studies into two groups was carried out based on the associated allele (group A: $C$ versus $G$ allele in IA patients versus control in $C$ allele-associated population (Fig. 4) and group B: G versus $\mathrm{C}$ allele in IA patients versus control in $\mathrm{C}$ alleleassociated population) (Fig. 5). This was specifically done as $\mathrm{G}$ allele was associated in Han Chinese ethnicity while $\mathrm{C}$ allele was found to be associated in Caucasian and Cantonese ethnicity. Association was observed with both $\mathrm{C}$ allele $(Z=2.38, p=0.02)$ and $\mathrm{G}$ allele $(Z=6.60$, $\mathrm{p}<0.00001$ ) with fixed effect model (Figs. 4 and 5). Meta- analysis with rs3212227 of $I L 12 B$ and rs16944 of $I L 1 B$ did not show any association in both fixed effect and random effect models (Figs. 6 and 7).

\section{Discussion}

The present study explores the genetic liability to inflammatory response induced by genetic variability in cytokine genes and how this genetic variability through differential inflammatory response could result in the development of aneurysm. The study comprised of proinflammatory cytokines IL1, IFNG, TNFA, and IL6; antiinflammatory cytokines ILIRN, TGFB1, and IL10; and pleiotropic cytokines $I L 3, I L 12 B$, and $I L 4$ genes. We report a novel association with pro-inflammatory cytokines

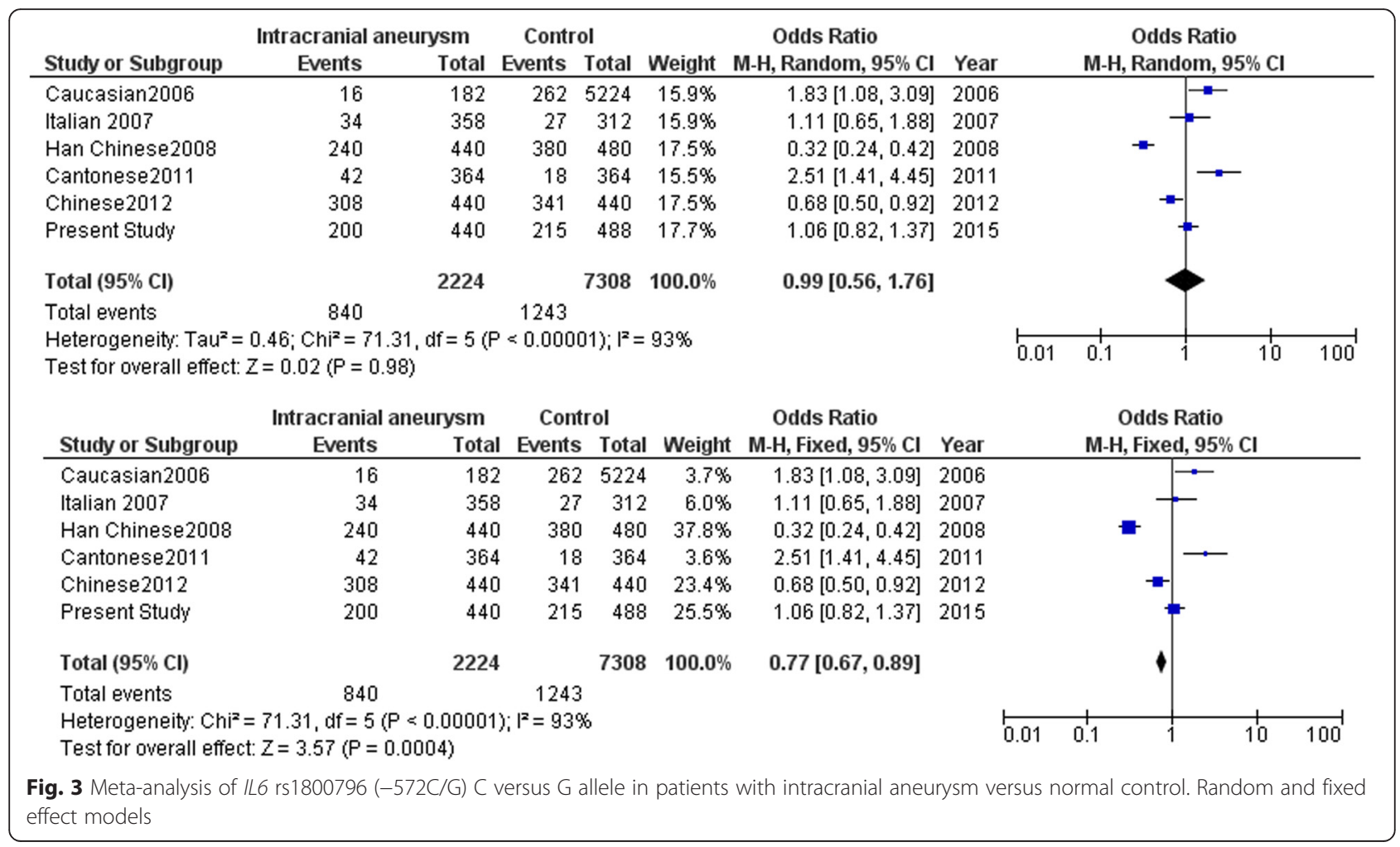




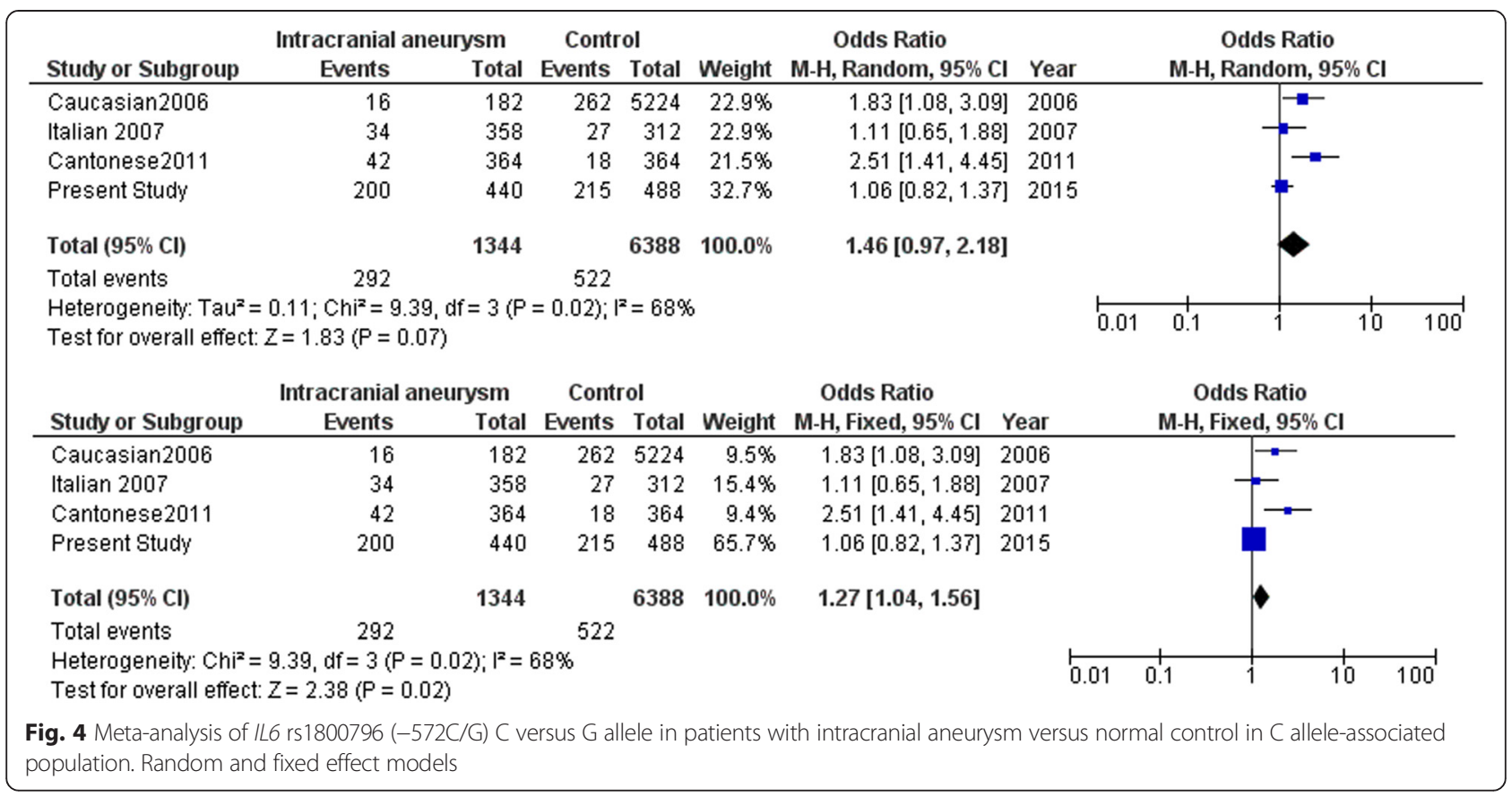

TNFA and IFNG. Interestingly, some of the reported association with the pro-inflammatory cytokines of IL1 gene cluster and IL6 were not found to be associated in the present study. None of the pleiotropic cytokines were associated with IA in the present study, but we did observe a novel association with anti-inflammatory cytokines $I L 10$. Most of the studies till date were restricted to individual cytokines and mainly focused on IL1B, IL6, and TNFA which could not be replicated in different ethnicities. We could not find any significant association for subgroup analysis which suggests that the associated SNPs might have a direct effect on the development of IA and was independent of hypertension and gender. Limitation of the study was with large effect size, and this would involve increased sample size. Large effect sizes are uncommon in complex diseases and therefore multiple testing was not applied to the study. Increasing sample size would mean sampling genetically non-stratified sample which will

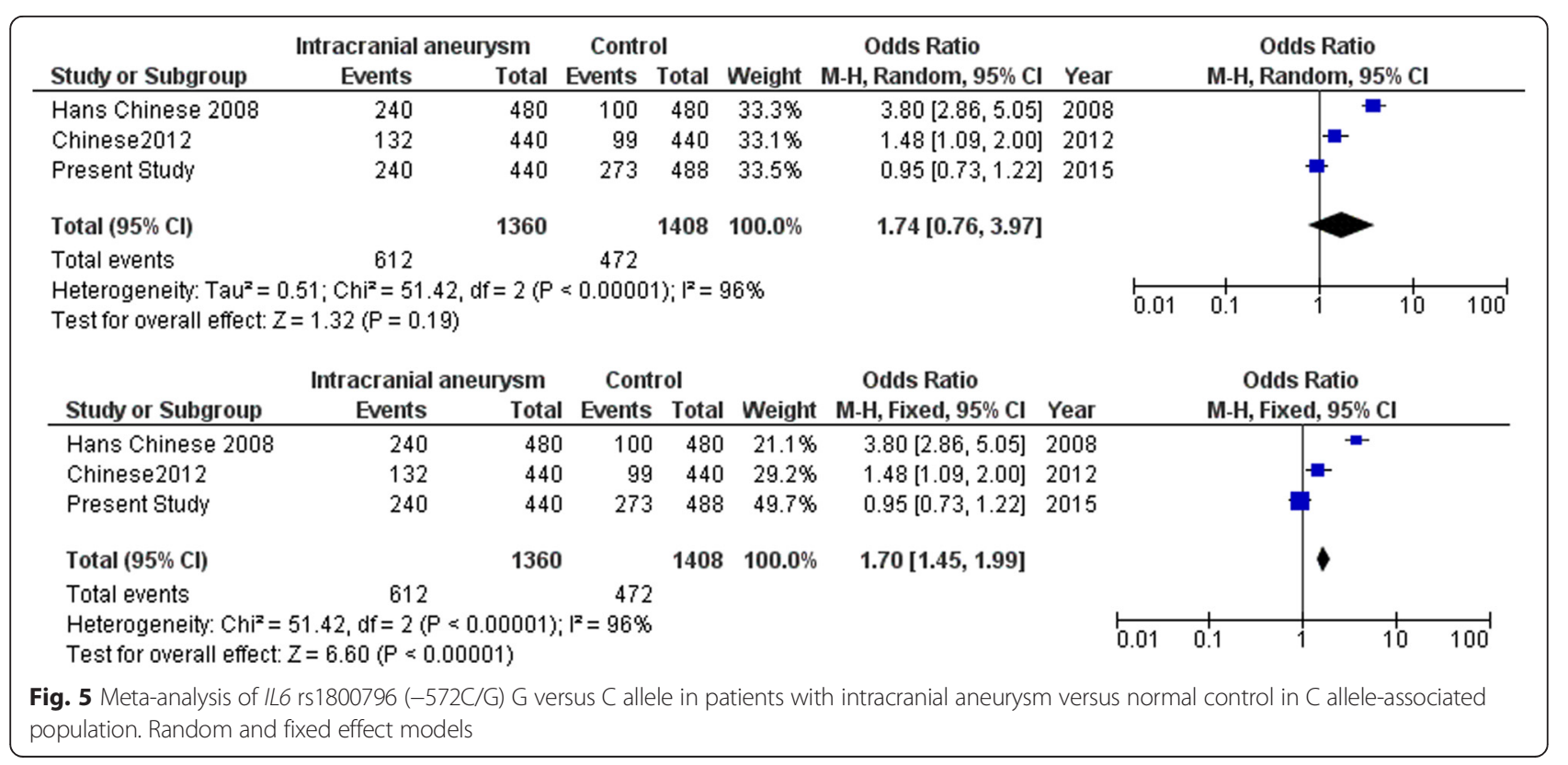




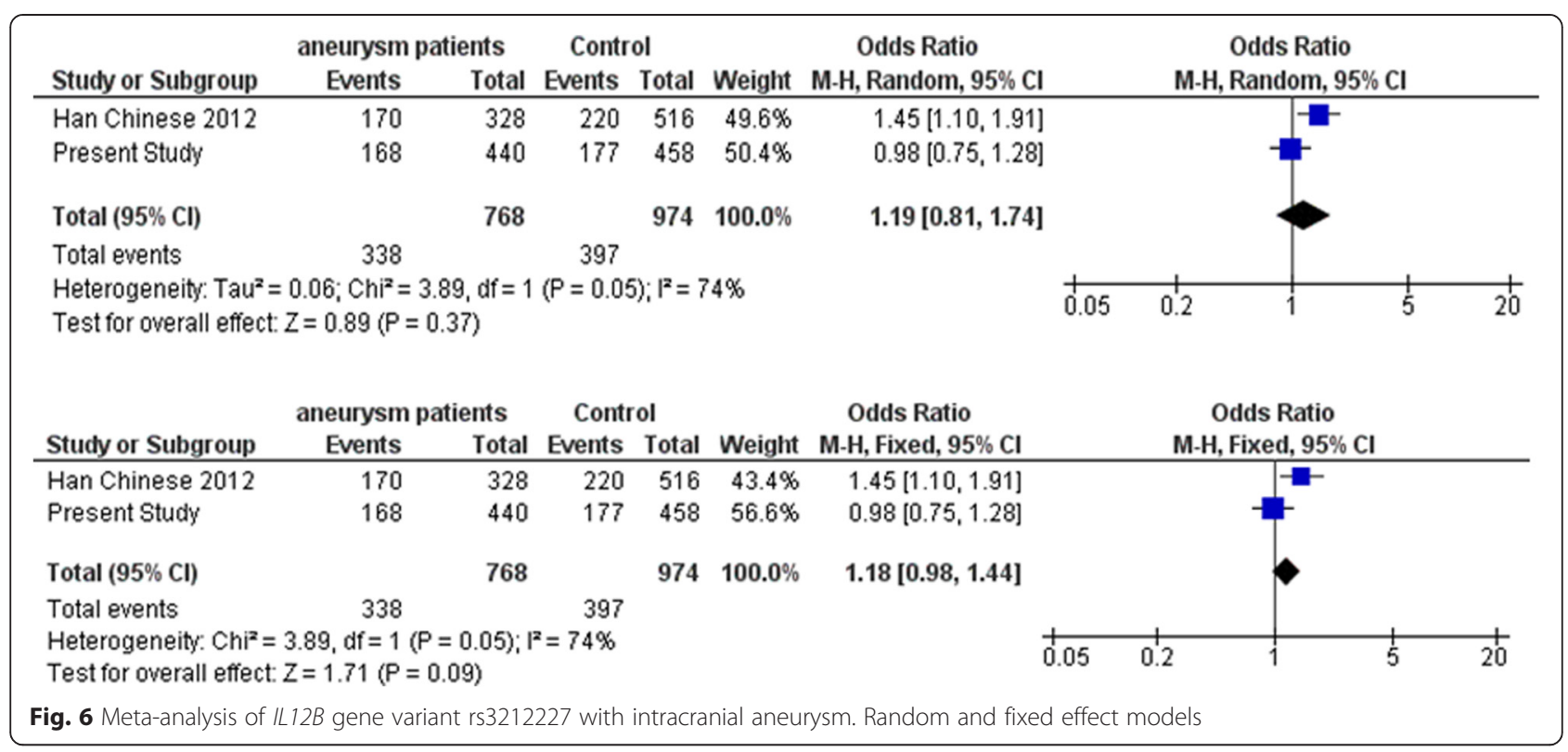

further compromise the observation. This is also the reason why genetic studies are not replicable across ethnicities, and this conflict is evident in the meta-analysis due to genetic heterogeneity. Monitoring the cytokine levels of these associated cytokines might be of interest in IA pathogenesis as the immunogenetic background might suggest an individual's response to a given immunological stress in the development and progression of aneurysm. The differential inflammatory response mediated by genetic variants in cytokines could be a primary event, while inflammatory response mediated by pre- or posttraumatic stress or comorbid factors could further augment the disease pathology.

Association with TNFA polymorphic variant in the present study is interesting as it is known to be a powerful pro-inflammatory cytokine. Expression studies indicate overexpression of TNFA to modulate susceptibility to IA [18]. While IA is known to be a multifactorial

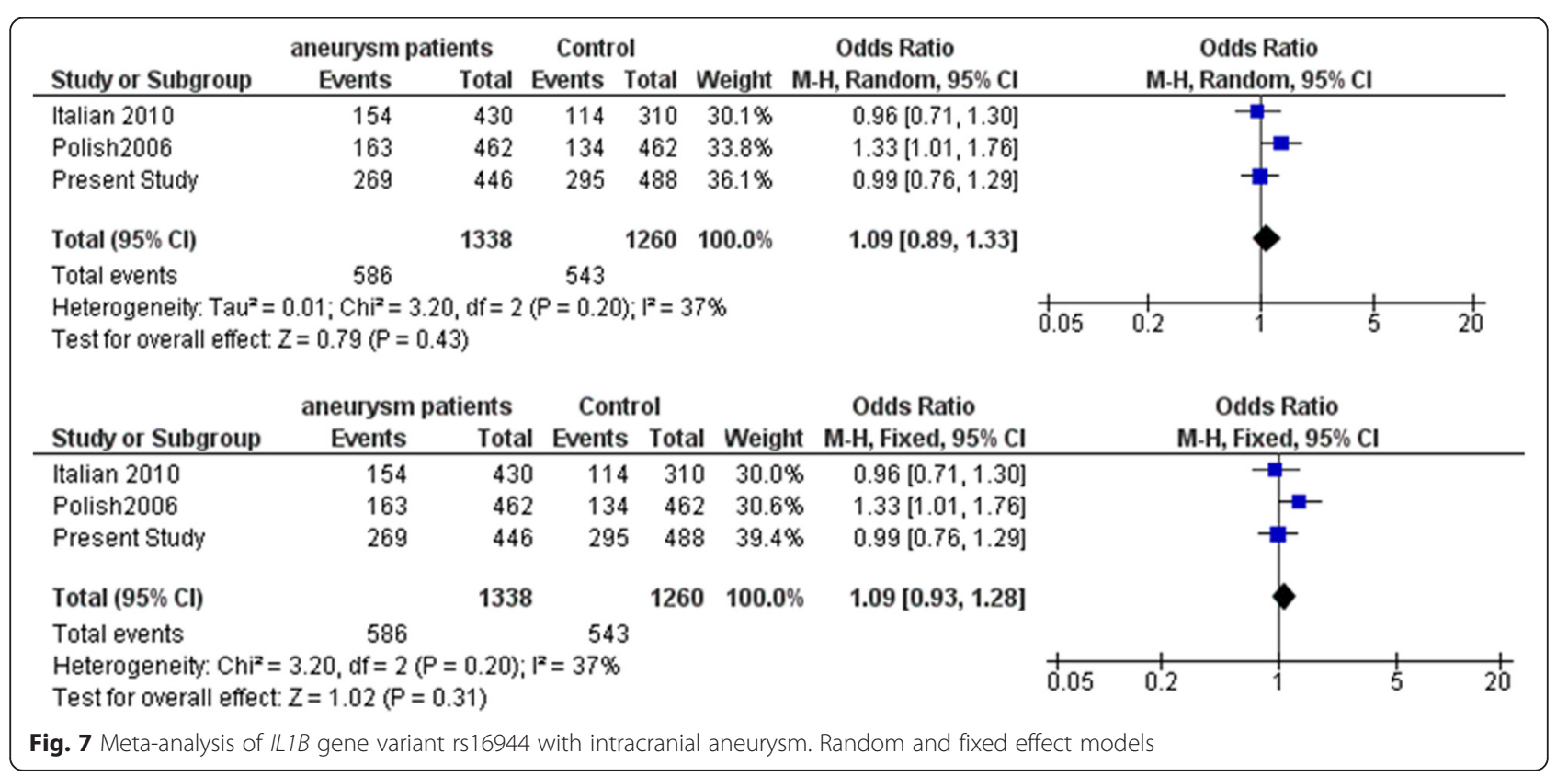


disease, similarly, the production and modulatory role of TNF is also multistimulatory. Therefore, how TNFA promotes pathogenesis of IA is indeed a challenge. In-Silico functional prediction suggests that rs361525 variant is involved in transcriptional regulation with FS score of 0.208 . Functional studies with rs 361525 show differential effect of transcriptional activation with TNFA rs361525238A allele conferring increased transcriptional activation of the TNF promoter [19]. "A" allele of rs361525 has been reported to enhance the responsiveness of TNFA promoter by 2.2 to 2.8 times greater versus the "G" variant, when stimulated with pro-inflammatory stimuli [19]. Functional validation studies further suggests that "A" allele of rs361525 creates a functionally active thyroid hormone receptor (TR) binding site which resulted in TR- $\alpha$-induced promoter overexpression by 1.8-fold. Earlier studies on TNFA provided evidence for rs1800629 ( $-308 \mathrm{G}<\mathrm{A})$ with IA in Italian population, but this SNP was not found to be associated with our study population [20]. It has been reported that among TNFA promoter polymorphisms, rs361525 could modulate transcriptional regulation but not the rs1800629 [21]. Therefore, association with " $\mathrm{A}$ " allele of rs361525 in the present study could indicate overproduction of TNF $\alpha$ as a genetic event and thereby mediating development and rupture of IA in south Indian population. Risk factors that associate with intracranial aneurysm such as hemodynamic stress [22], hypertension [23], and smoking [24] are also known to induce the production of TNFo. Increased stimulation of TNF $\alpha$ and Fasassociated death domain protein, which are involved in inflammation and apoptosis, can have deleterious effect on arterial wall [18]. Thus, in the present study, the over representation of $\mathrm{A}$ allele which refers to increased transcriptional activation of TNF promoter may result in the development of aneurysm or aneurismal rupture, through its multistimulatory role induced by multifactorial nature of IA.

Genetic association of TNFA and IFNG may further exacerbate the pro-inflammatory role as TNFA is known to synergize with IFNG in inhibiting the proliferation of various cell types. The growth inhibitory activities of IFNG are more pronounced in endothelial cells. Polymorphisms in the promoter regions of IFNG have been reported to influence IFN- $\gamma$ production. In the present study, we report higher representation of IFNG +3234TT genotype in aneurismal patients. Based on the functional studies of $+3234 \mathrm{C} / \mathrm{T}$ polymorphism, $+3234 \mathrm{C}$ alleles has been reported to be associated with a higher level of IFN$\gamma$ than the $+3234 \mathrm{~T}$ alleles [25]. Higher frequency of homozygous $+3234 \mathrm{TT}$ genotype could be indicative of lower levels of IFN- $\gamma$. The regulation of IFNG gene transcription is complex and several transcription factors have been found to interact with the proximal promoter constitutively [26] or by induction [27]. One of the most important functions of IFNG is macrophage activation function, which upregulates the expression of the major histocompatibility complexes (MHC)I [28] and MHCII [29], involved in antigen processing and presentation pathways. IFN- $\gamma$ also mediates functions such as leukocyte attraction [28], maturation and differentiation, natural killer (NK) cell activity [30], and immunoglobulin (Ig) production and class switching in B cells [28].Therefore, reduced IFN- $\gamma$ production would result in compromised resultant functions such as reduced leukocyte attraction or macrophage activation.

Pro-inflammatory cytokines are linked in a sequence resembling an electrical circuit in series, with TNF- $\alpha$ at the apex of the cascade inducing production of IL-1, and subsequently both TNF- $\alpha$ and IL-1 induce downstream cytokines such as IL-6 [31]. Polymorphisms in any of these genes are likely to alter the downstream functional events. Earlier studies did provide evidence with contradictions for involvement of IL1 gene complex with IA. A study in Polish population has reported an association of $I L 1 B$ rs 16944 with IA, but this could not be replicated in Italian population [16, 17]. IL1RN VNTR polymorphism has been widely implicated in ischemic stroke [32] and coronary artery disease (CAD) [33]. In the present study, we failed to replicate these observations in IA patients from south India. Another pro-inflammatory cytokine IL6 had been the point of intense study by several investigators. Morgan et al. (2006) first reported that IL6 promoter polymorphism rs1800796 $(-572 \mathrm{C} / \mathrm{G})$ and rs1800795 $(-174 \mathrm{G} / \mathrm{C})$ are associated with IA in Caucasian population and 572C/ 174C haplotype having a greater risk [10]. Subsequent studies with these variants in Italian and Chinese population had shown conflicting results [11-14]. While rs1800796 and rs1800795 were not associated with IA in Italian population [11], it was a conflict of allelic associations in Chinese population. In Han Chinese [12] and west Chinese population [14], G allele proved to be a risk allele, whereas in Cantonese population, $\mathrm{C}$ allele turned out to be a risk allele for IA [13]. None of these allelic associations for IL6 could be replicated in our study population for IA. Observations from some of these extensively investigated proinflammatory cytokines remained inconclusive; therefore, we carried out a meta-analysis with these SNPs by including the present study. Meta-analysis with rs3212227 of $I L 12 B$ and rs16944 of $I L 1 B$ did not show any association in both fixed effect and random effect models, while with IL6 indicated tremendous heterogeneity between studies which could be accounted to large variation in different studies shown by opposite alleles being associated in different world population. Therefore, conflicting allelic association of IL6 needs to be carefully interpreted in 
terms of its functional significance to environment and comorbid factors that can influence IL6 production and subsequently result in development of IA. These ethnic-specific genetic associations are important to understand the complex nature of the disease which might help in modeling the gene-environment interaction. All these studies indicate that the cascade of events in pro-inflammatory cytokine circuit is active, but sequence of events in the circuit tends to differ in different ethnicities in developing risk for IA.

None of the anti-inflammatory or pleiotropic cytokines were found to be associated at allelic or genotypic combinations in the present study with exception to IL10. In IL10, the heterozygous genotypes of the two tagged SNPs rs1800871 and rs1800872 were observed to be associated with IA. IL10 is a potent antiinflammatory and immunomodulatory cytokine, exerting major effects in the degree and quality of the immune response. It is a potent suppressor of proinflammatory cytokines such as IL1, IL6, IL12, TNFA, and IFNG and inhibits the costimulatory activity of macrophages for $\mathrm{T}$ cell and NK cell activation. It is monoallelically expressed in CD4+ T cells. Evidences suggest that the monoallelic expression pattern of IL1O is not due to parental imprinting, allelic exclusion, or strong allelic bias, instead, due to stochastic regulation mechanism, in which the probability to initiate allelic transcription depends on the strength of TCR signaling and subsequent capacity to overcome restrictions imposed by chromatin hypoacetylation. Allelic expression data shows transcriptional independence between both alleles [34]. This could possibly help us understand why in an earlier study, IL1O was not found to express in IA patients [18]. Monoallelic expression and stochastic regulation of IL10 might help us understand the functional implication of heterozygous genotype with IAs, observed in the present study. The rs1800872 variant has been reported to be a low-producer allele of the IL10 gene [35]. A recent study have reported that approximately one third of the genes have the same allele expressed more highly in all heterozygotes, suggesting that their regulation is predominantly influenced by cis elements in strong linkage disequilibrium with the assayed exonic SNP. The remaining two thirds of the genes have different alleles expressed more highly in different heterozygotes, suggesting that their expression differences are influenced by factors not in strong linkage disequilibrium with the assayed exonic SNP [36]. Interestingly, stochastic monoallelic expression of IL10 has been reported which are transcriptionally independent [34]. Based on these observations, one can suggest that the pathologic modulation of cis- or the trans-acting factors on the heterozygous genotypes might result in the development of aneurysm.

\section{Conclusion}

We conclude that genetic liability of anti-inflammatory IL10 rs1800871 and rs1800872 variant in combination with pro-inflammatory TNFA rs361525 and IFNG rs2069718 gene variant may promote pathogenesis of IA. The differential inflammatory response mediated by genetic variants in cytokines could be a primary event, while inflammatory response mediated by stochastic events of pre- or posttraumatic stress or comorbid factors could further augment the disease pathology. It has been reported that locally secreted cytokines may tilt the regional balance of matrix metalloproteinases (MMP) activity in favor of vascular matrix degradation [37]. Therefore, chronic exposure to pro-inflammatory cytokines TNFA and IFNG and stochastic regulation of IL10 and TGFB in response to pathologic environment may support development of IA. The implication and interaction of these genetic variants under a specific environmental background will help us identify the resultant phenotypic variation in the pathogenesis of intracranial aneurysm. Identifying genetic risk factors for inflammation might also help in understanding and addressing the post-traumatic complications following the aneurismal rupture.

\section{Additional file}

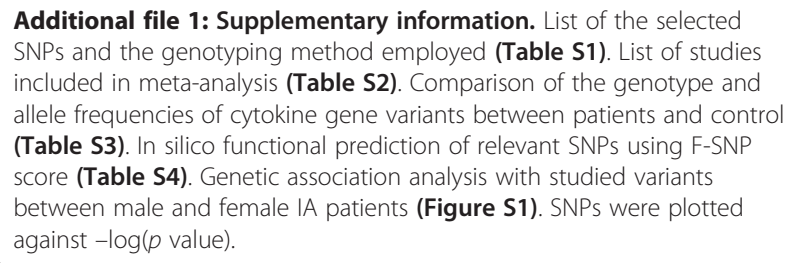

\section{Competing interests}

The authors declare that they have no competing interests.

\section{Authors' contributions}

SS and LS carried out the genotyping and sequencing work. SS and LKV were involved in sample collection and genomic DNA screening from patients. HVE, PS, JA, and SN were involved in patient characterization and clinical profiling. RNB and MB conceptualized and designed the work. MB and SS carried out the statistical analysis of the genetic design, while RNB and SN coordinated the clinical part of the study. All authors read and approved the final manuscript.

\section{Acknowledgments}

SS acknowledges the Council of Scientific and Industrial Research (CSIR), Government of India, for providing junior research fellowship. MB acknowledges the intramural funding for carrying out the work.

\section{Author details}

${ }^{1}$ Human Molecular Genetics Laboratory, Rajiv Gandhi Centre for Biotechnology, Thiruvananthapuram 695014Kerala, India. ${ }^{2}$ Department of Neurosurgery, Sree Chitra Tirunal Institute for Medical Science and Technology, Thiruvananthapuram, Kerala, India. ${ }^{3}$ Department of Neurosurgery, Calicut Medical College, Calicut, Kerala, India.

Received: 5 May 2015 Accepted: 29 June 2015

Published online: 22 July 2015 


\section{References}

1. Bederson JB, Awad IA, Wiebers DO, Piepgras D, Haley Jr EC, Brott T, et al. Recommendations for the management of patients with unruptured intracranial aneurysms: a statement for healthcare professionals from the Stroke Council of the American Heart Association. Circulation. 2000:102:2300-8

2. van Gijn J, Kerr RS, Rinkel GJ. Subarachnoid haemorrhage. The Lancet. 2007;369:306-18

3. Koshy L, Easwer H, Premkumar S, Alapatt JP, Pillai AM, Nair S, et al. Risk factors for aneurysmal subarachnoid hemorrhage in an Indian population. Cerebrovasc Dis. 2010;29:268-74.

4. Woo D, Broderick J. Genetics of intracranial aneurysm. Elsevier. 2002;2:24-34.

5. Meng H, Wang Z, Hoi Y, Gao L, Metaxa E, Swartz DD, et al. Complex hemodynamics at the apex of an arterial bifurcation induces vascular remodeling resembling cerebral aneurysm initiation. Stroke. 2007;38:1924-31.

6. Chyatte D, Bruno G, Desai S, Todor DR. Inflammation and intracranial aneurysms. Neurosurgery. 1999;45:1137.

7. Krischek B, Kasuya H, Tajima A, Akagawa H, Sasaki T, Yoneyama T, et al. Network-based gene expression analysis of intracranial aneurysm tissue reveals role of antigen presenting cells. Neuroscience. 2008;154(4):1398-407.

8. Slifka MK, Whitton JL. Clinical implications of dysregulated cytokine production. J Mol Med. 2000;78:74-80.

9. Thomas R, Nair S, Banerjee M. HLA-B and HLA-C alleles and haplotypes in the Dravidian tribal populations of southern India. Tissue Antigens. 2004;64:58-65.

10. Morgan L, Cooper J, Montgomery H, Kitchen N, Humphries SE. The interleukin-6 gene-174G>C and-572G>C promoter polymorphisms are related to cerebral aneurysms. J Neurol Neurosurg Psychiatry. 2006;77:915-17

11. Fontanella M, Rainero I, Gallone S, Rubino E, Fenoglio P, Valfrè W, et al Interleukin 6 gene polymorphisms are not associated with aneurysmal subarachnoid haemorrhage in an Italian population. J Neurol Neurosurg Psychiatry. 2008;79:471-3.

12. Sun H, Zhang D, Zhao J. The interleukin-6 gene-572G>C promoter polymorphism is related to intracranial aneurysms in Chinese Han nationality. Neurosci Lett. 2008;440(1):1.

13. Zhang G, Tu Y, Feng W, Huang L, Li M, Qi S. Association of interleukin-6$572 \mathrm{G} / \mathrm{C}$ gene polymorphisms in the Cantonese population with intracranial aneurysms. J Neurol Sci. 2011;306:94-7.

14. Liu Y, Sun J, Wu C, Cao X, He M, You C. The interleukin-6-572G/C gene polymorphism and the risk of intracranial aneurysms in a Chinese population. Genet Test Mol Biomarkers. 2012;16:822-6.

15. Li L-J, Pan X-M, Sima X, Li Z-H, Zhang L-S, Sun H, et al. Interactions of interleukin-12A and interleukin-12B polymorphisms on the risk of intracranial aneurysm. Mol Bio Reports. 2012;39:11217-23.

16. Fontanella M, Rainero I, Gallone S, Rubino E, Fornaro R, Fenoglio P, et al. Interleukin-1 cluster gene polymorphisms and aneurysmal subarachnoid hemorrhage. Neurosurgery. 2010;66:1058-63.

17. Slowik A, Borratynska A, Turaj W, Pera J, Dziedzic T, Wloch D, et al. Interleukin $1 \beta-511 \mathrm{C} / \mathrm{T}$ polymorphism and risk of aneurysmal subarachnoid haemorrhage. J Neurol Neurosurg Psychiatry. 2006;77:279.

18. Jayaraman T, Berenstein V, Li X, Mayer J, Silane M, Shin YS, et al. Tumor necrosis factor a is a key modulator of inflammation in cerebral aneurysms. Neurosurgery. 2005;57:558-64.

19. Kiss-Toth E, Harlock E, Lath D, Quertermous T, Wilkinson JM. A TNF variant that associates with susceptibility to musculoskeletal disease modulates thyroid hormone receptor binding to control promoter activation. PLoS One. 2013;8, e76034.

20. Fontanella M, Rainero I, Gallone S, Rubino E, Fenoglio P, Valfrè W, et al. Tumor necrosis factor-a gene and cerebral aneurysms. Neurosurgery. 2007;60:668-73.

21. Bayley J-P, de Rooij H, van Den Elsen PJ, Huizinga TW, Verweij CL. Functional analysis of linker-scan mutants spanning the-376,-308,- 244, and- 238 polymorphic sites of the TNF-a promoter. Cytokine. 2001;14:316-23.

22. Gonzalez CF, Cho Yl, Ortega HV, Moret J. Intracranial aneurysms: flow analysis of their origin and progression. Amer J Neuroradiol. 1992;13:181-8.

23. Muñoz J, Albillos A, Pérez-Páramo M, Rossi I, Alvarez-Mon M. Factors mediating the hemodynamic effects of tumor necrosis factor-a in portal hypertensive rats. Am J Physiol. 1999;276(3 Pt 1):G687-G93.
24. Sun S, Tang W, Liu C, Lin H, Yang H. Effects of tumor necrosis factor alpha on proteolysis of respiratory muscles in rats with chronic obstructive pulmonary disease. Zhonghua Jie He He Hu Xi Za Zhi. 2007;30:186-91.

25. Huang $Y$, Yang $H$, Borg BB, Su X, Rhodes SL, Yang $K$, et al. A functional SNP of interferon- $\gamma$ gene is important for interferon-a-induced and spontaneous recovery from hepatitis C virus infection. Proc Natl Acad Sci U S A 2007; 104:985-90

26. Ye J, Cippitelli M, Dorman L, Ortaldo JR, Young HA. The nuclear factor YY1 suppresses the human gamma interferon promoter through two mechanisms: inhibition of AP1 binding and activation of a silencer element. Mol Cell Biol. 1996;16:4744-53.

27. Kojima H, Aizawa Y, Yanai Y, Nagaoka K, Takeuchi M, Ohta T, et al. An essential role for NF-KB in IL-18-induced IFN- $\gamma$ expression in KG-1 cells. J Immunol. 1999;162:5063-9.

28. Boehm U, Klamp T, Groot M, Howard J. Cellular responses to interferon- $\gamma$. Ann Rev Immunol. 1997;15:749-95.

29. Chang $\mathrm{C}-\mathrm{H}$, Flavell RA. Class II transactivator regulates the expression of multiple genes involved in antigen presentation. J Exp Med. 1995;181:765-7.

30. Carnaud C, Lee D, Donnars O, Park S-H, Beavis A, Koezuka Y, et al. Cutting edge: cross-talk between cells of the innate immune system: NKT cells rapidly activate NK cells. J Immunol. 1999;163:4647-50.

31. Maini RN, Elliott MJ, Brennan EM, Williams RO, Chu CQ, Paleolog E, et al. Monoclonal anti-TNFa antibody as a probe of pathogenesis and therapy of rheumatoid disease. Immunol Rev. 1995;144:195-223.

32. Worrall BB, Brott TG, Brown RD, Brown WM, Rich SS, Arepalli S, et al. IL1RN VNTR polymorphism in ischemic stroke analysis in 3 populations. Stroke. 2007;38(4):1189-96.

33. Arman A, Soylu O, Yildirim A, Furman A, Ercelen N, Aydogan H, et al. Interleukin-1 receptor antagonist gene VNTR polymorphism is associated with coronary artery disease. Arq Bras Cardiol. 2008;91:293-8.

34. Calado DP, Paixão T, Holmberg D, Haury M. Stochastic monoallelic expression of IL-10 in T cells. J Immunol. 2006;177:5358-64.

35. Ding $Q$, Shi $Y$, Fan B, Fan Z, Ding L, Li F, et al. The interleukin-10 promoter polymorphism rs $1800872(-592 \mathrm{C}>\mathrm{A})$, contributes to cancer susceptibility: meta-analysis of 16785 cases and 19713 controls. PLoS One. 2013;8(2), e57246.

36. Pant PK, Tao H, Beilharz EJ, Ballinger DG, Cox DR, Frazer KA. Analysis of allelic differential expression in human white blood cells. Genome Res. 2006;16:331-9.

37. Galis ZS, Muszynski M, Sukhova GK, Simon-Morrissey E, Unemori EN, Lark MW, et al. Cytokine-stimulated human vascular smooth muscle cells synthesize a complement of enzymes required for extracellular matrix digestion. Circ Res. 1994;75:181-9.

\section{Submit your next manuscript to BioMed Central and take full advantage of:}

- Convenient online submission

- Thorough peer review

- No space constraints or color figure charges

- Immediate publication on acceptance

- Inclusion in PubMed, CAS, Scopus and Google Scholar

- Research which is freely available for redistribution 